\title{
Research on The High-speed Sewing Control Technology for the Electronic Pattern Sewing Machine
}

\author{
Lu Feng ${ }^{1}$, Gong Shihua ${ }^{1}$, Gu Xiqian ${ }^{2}$, Huang $\mathrm{Yu}^{1}$, Wang Pingjiang ${ }^{1}$, Shao Wenjun ${ }^{1}$, Guo Xin ${ }^{1}$ \\ 1. State Engineering Research Center of Manufacturing Equipment Digitization, Huazhong University of Science and \\ Technology, Wuhan 430074, Hubei, China \\ 2. Military economics academy, Wuhan 430074, Hubei, China
}

\begin{abstract}
Based on the control requirement for high speed of the electronic pattern sewing machine, the architecture of open control system, in which based on an ARM embedded system is used for human machine interface and other nonreal-time tasks and motion controller is used for servo control and logic control etc, is proposed to control stitching process with motion controller and Ethernet. The velocity planning of cloth frame and the spindle speed arithmetic are researched. Then the time-optimal control principle is proposed to scheme the frame velocity curve and the minimum movement time. Test Results show that it can reach 2800 stitches per minute, which is the international level.
\end{abstract}

Keywords- Electronic pattern sewing machine, High-speed sew, Spindle speed, Cloth frame motion control

\section{INTRODUCTION}

The Demand of the intelligent industrial sewing machine performs exponential growth at present. However, domestic research and development starts late, and technical Level is low, so the overall yield is far less than the market demand. As the top product in the intelligent industrial sewing machine industry, the electronic pattern sewing machine has great prospects for development.

High-speed is an important index to evaluate the quality of the machine. The international advanced level in the Field of the Electronic Pattern Sewing Machine is 2800 stitches per minute at highest speed. Meanwhile, the machine made in china only reaches 2400 stitches per minute. There is still much difference between our country's application and the international level. The ARM unit under Microsoft Windows CE Operation System can communicate

with the motion controller timely in the system, and the control of the stitching motion is programmed with advanced language under the $\mathrm{NC}$ unit. By using the control technology related in this paper, the stitching speed can achieve 2813.6 stitches per minute, which has reached international level.

\section{HARDWARE CONFIGURATION OF THE CONTOL SYSTEM}

The system of ARM unit and NC unit is used to realize stitch control. Pattern formation, task communication and memory management are mainly done on the ARM unit which based on an ARM embedded system. As the NC unit, the motion controller controls sewing path movement, realizes auxiliary functions such as cutting thread and pause. Communication between the ARM unit and NC unit is based on the TCP/IP protocol in industrial Ethernet. The designed hardware structure diagram is shown in Figure1.
There are three motors controlled by the motion controller, two step motors are used to drive $\mathrm{X}-\mathrm{Y}$ table move where cloth is pressed, and the servo motor is the spindle. The axisU rotary motion is converted to needles' up-down repeated movement by the crank and connecting rod mechanism. Moreover, the motion controller provides users with IO ports, which are used to receive users' information, send control commands and show the system state such as fault and pause.

The stitching motion is programmed with advanced language under the $\mathrm{NC}$ unit, which receive the information such as stitch data and parameters from the ARM unit, control the spindle and XY step motors' motion in harmony. The measures listed below are taken to ensure the high speed.

The information is transferred rapidly based on the communication between the ARM unit and $\mathrm{NC}$ unit

Every axis is controlled by the NC unit timely, so the equipments can response rapidly.

\section{Motion CONTROL OF THE High-SPEED StITCHING}

The Stitch data generated in the ARM unit are downloaded into $\mathrm{NC}$ unit in the form of data program. In order to transfer the whole information, the data mainly consist of three data arrays, X_Pos, Y_Pos and M. The former two data arrays store the position of every stitch, and the last one is used to store the other information, such as the pause command. Several bits are combined to record the information in $\mathrm{M}$ data array, in this way, the data file can be reduced largely and the download speed is raised. Take bit 1-3 as an example, there is no code data when the combination of these tree bit is 000 , and 001 means uppause. The information can be got by combining the bits and checking in table. 1 in the motion controller.

TABLE I. DEFINITION OF THE DATA ARRAYS

\begin{tabular}{|l|l|l|}
\hline $\begin{array}{l}\text { Bits (low } \\
\text { pos first) }\end{array}$ & \multicolumn{1}{|c|}{ Definition } & Description in Detail \\
\hline 0 & $\begin{array}{l}\text { Referral or } \\
\text { stitch }\end{array}$ & 0: Referral 1: Stitch \\
\hline $1-3$ & Code data & $\begin{array}{l}\text { 000:No code 001:Up-pause 010: } \\
\text { Down-pause 011:Cut thread }\end{array}$ \\
\hline $4-5$ & Graph speed & $\begin{array}{l}\text { 00:Low speed 01:Middle-low speed } \\
\text { 10:Middle-high speed 11:High speed }\end{array}$ \\
\hline $6-17$ & $\begin{array}{l}\text { Speed limited } \\
\text { by stitch } \\
\text { length }\end{array}$ & $\begin{array}{l}\text { Only valid when the graph } \\
\text { speed is high }\end{array}$ \\
\hline
\end{tabular}

There are several modes to control the stitch motion. 
Upper-control mode: In this control mode, all commands are sent by ARM unit, transferred to the NC unit based on the TCP/IP protocol in industrial Ethernet, interpreted and executed. Due to the accumulated time error, the time between sending commands and performing commands would be so long that the users' commands can't be responded rapidly and the stitch speed is hard to rise.

Lower-control mode: When the commands are sent by the NC unit, it's called lower control mode. G code control mode and defined-command control mode are two commonly-used modes. The former one adopted three axes (two step motors and one servo motor) interpolation to realize control. The step speed limits the stitch speed ultimately. The control program is in the NC unit in the defined-command control mode. The machine can receive messages sent by the users through IO ports, and send command to the equipment rapidly. The servo motor (axis-U) and step motors are controlled independently to raise the stitch speed. The step motors' motion is triggered by the preset position of the axis- $U$. The second one is adopt, which is shown in Figure 2.

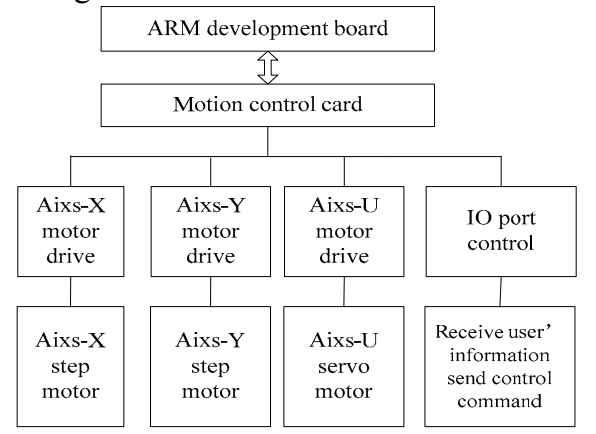

Figure 1. Hardware structure diagram

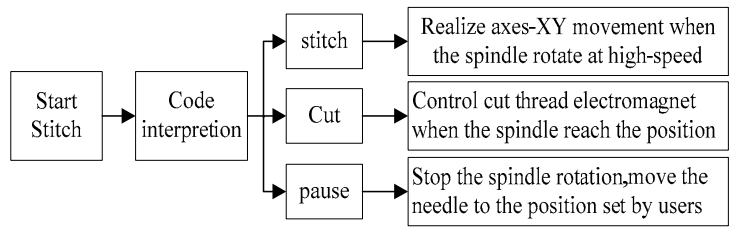

Figure 2. Stitch control flow chart

\section{A. OPTITMIZATION OF THE SPINDLE ROTATING SPEED}

The formula below is a commonly used traditional algorithm in the control system of electronic pattern sewing machine[1].

$$
\left\{\begin{array}{lrl}
n_{0}=n_{N} & \left(0 \leq l \leq l_{0}\right) \\
n_{0}=n_{N}-\frac{\left(n_{N}-n_{\min }\right)\left(l-l_{0}\right)}{l_{\max }-l_{0}} & \left(l_{0}<l \leq l_{\max }\right)
\end{array}\right.
$$

Where $\mathrm{nN}$ is the spindle rotating speed when the stitch length is typical. 10 is the critical stitch length (spindle rotating speed should be cut down when the stitch length longer than this value) and nmin is the lowest rotating speed of the spindle when the stitch length is the longest one.
Research shows that relation table of the stitch length and spindle rotating speed can be established according to the corresponding relation between the typical stitch length and spindle rotating speed in condition of ensuring the trajectory precision of axes-XY. Assuming the speed is $\mathrm{n} 1$ when the stitch length is 11 , and speed $\mathrm{n} 2$ is corresponding to 12 , the equation of the spindle rotating speed algorithm is given by

$$
\left\{\begin{array}{lc}
n_{0}=n_{N} & \left(0 \leq l \leq l_{0}\right) \\
n_{0}=n_{2}-\frac{\left(n_{2}-n_{1}\right)\left(l-l_{1}\right)}{l_{2}-l_{1}} & \left(l>l_{0}, l \in\left[l_{1}, l_{2}\right]\right)
\end{array}\right.
$$

The comparison of two algorithms is shown in Figure3, while the first one is the traditional algorithm and the other is the new one. The curve in the chart shows that the second algorithm is better than the first one at the point of promoting the spindle speed, especially the stitch length is long.

\section{B. motion control of the high_speed cloth frame}

In the condition of ensuring spindle's high rotating speed, the cloth frame should be moved to the destination in set time in order to realize the high speed of the machine. Assuming the spindle rotating speed is $\mathrm{n} 0(\mathrm{r} / \mathrm{min})$, and then the time of one stitch is:

$$
\begin{aligned}
& T_{0}=\frac{60}{n_{0}}(s) \\
& n_{0} \leq \frac{60 \eta}{t_{m}}(\mathrm{r} / \mathrm{min})
\end{aligned}
$$

Due to the short time $(10 \mathrm{~ms})$ of the cloth frame's movement, acceleration and deceleration curve is designed in Figure 5 in order to reach the high moving speed. In the Figure 5, time segment $\mathrm{t} 1 \mathrm{-t} 2$ and $\mathrm{t} 3-\mathrm{t} 4$ are the time when cloth frame move, and T0 is the period of the needle rotates one cycle. The cloth frame can reach the highest speed if it moves according the rule shown in Figure5. The acceleration and load torque are calculated:

$$
\begin{aligned}
& a=\frac{2 s}{t_{m}^{2}} \geq \frac{2 s}{\left(\eta T_{0}\right)^{2}} \\
& s=\frac{1}{2} a t_{m}^{2}
\end{aligned}
$$

The lowest acceleration can be calculated when the spindle speed is set at 2800 $3 \mathrm{~mm}$.

stitches per minute, $\eta$ equal to 0.5 and the stitch length is

$$
\begin{aligned}
& T_{0}=\frac{1}{n_{0}}=\frac{1 \times 60 \times 1000}{2800} \mathrm{~ms}=0.021 \mathrm{~s} \\
& a_{\min }=\frac{2 \mathrm{~s}}{\left(\eta T_{0}\right)^{2}}=\frac{2 \times 3}{(0.5 \times 0.021)^{2}} \mathrm{~mm} / \mathrm{s}^{2}=54421.77 \mathrm{~mm} / \mathrm{s}^{2}
\end{aligned}
$$


Load torque $T_{L}=\frac{9.55 \eta_{C}^{\prime} F v}{n_{M}}$

$$
\text { Motor torque } T_{M}=T_{L}+\frac{G D^{2}}{375} \frac{d n}{d t}=\frac{9.55 \eta_{C}^{\prime} F v}{n_{M}}+\frac{m g D^{2}}{375} \frac{d n}{d t}
$$

Where TL is the Load torque, TM is the motor torque, $\eta^{\prime} \mathrm{C}$ is the Transmission Efficiency, $\mathrm{F}$ is the load force, $\mathrm{v}$ is the speed, $\mathrm{nM}$ is the spindle speed, $\mathrm{m}$ is the load mass, $\mathrm{D}$ is the inertial radius, $t$ means time, then $\mathrm{dn} / \mathrm{dt}$ is the signed acceleration.

The cloth frame need start run and stop frequently, and the stop position requires high-precision in the system. Hybrid stepping motor is adopted according to these conditions. The selected step motor also should satisfy the conditions such as more than $54 \mathrm{~m} / \mathrm{s} 2$ acceleration, torquefrequency characteristic in equation 10 .

Subdivision technology is introduced in the driving of the step motors to ensure high positioning precision, decrease the starting torque and reduce the frequency resonance, prevent missing position of the step motor[2]. The driving figure of the step motor is shown in Figure6[3].

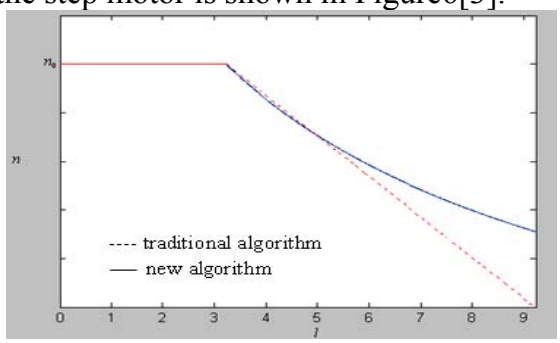

Figure 3. comparison chart between n0 -1

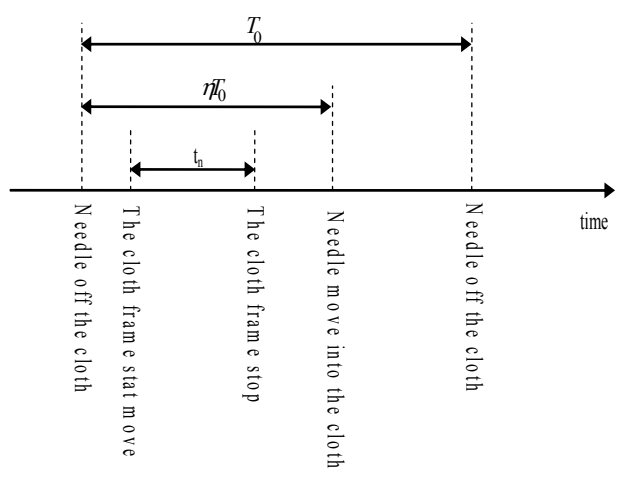

Figure 4. Sequence Diagram with the needle and cloth frame

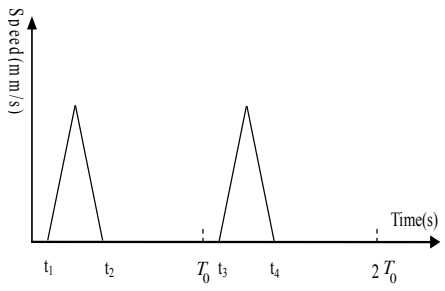

Figure 5. The speed curve of the cloth

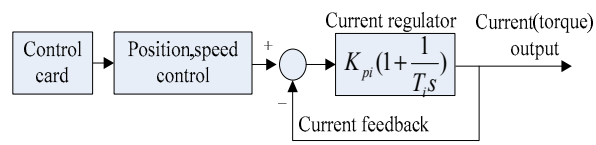

Figure 6. structure diagram of the cloth frame feed unit

\section{TESTING ANALYSIS AND CONCLUSION}

The motion controller is selected to control stitch motion as the control core. At last, the machine stitch stationarily with high speed and the product is beautiful, temper and smooth, which fully realized excellent performance of the electronic pattern sewing machine. In order to test the highest speed, the stitch length is set $3 \mathrm{~mm}$. Several testing data are shown in tab.2, calculating the average speed value is 2813.63 stitches per minute, which has exceeded the international advanced level (2800 stitches/min). The results show that the system referred in this paper has achieved international advanced level.

TABLE II. TESTING DATA OF THE SPEED

\begin{tabular}{|l|l|l|l|}
\hline \multicolumn{1}{|c|}{ Graph } & \multicolumn{1}{|c|}{$\begin{array}{c}\text { Total Stitches } \\
\text { (stitch) }\end{array}$} & \multicolumn{1}{|c|}{$\begin{array}{c}\text { Time } \\
(\mathbf{s})\end{array}$} & $\begin{array}{c}\text { Speed } \\
\text { (stitch/min } \\
\text { ) }\end{array}$ \\
\hline 1 & 514 & 10.97 & 2811.30 \\
\hline 2 & 734 & 15.62 & 2819.46 \\
\hline 3 & 820 & 17.51 & 2809.82 \\
\hline 4 & 953 & 20.31 & 2815.36 \\
\hline 5 & 1093 & 23.32 & 2812.18 \\
\hline $\begin{array}{l}\text { Average } \\
\text { Value }\end{array}$ & 823 & 17.55 & 2813.63 \\
\hline
\end{tabular}

\section{REFERENCES}

[1] GONG Shi-hua; HUANG Yu; ZHU Guo-li; LU Feng. Key technology of high speed shuttle embroidery machine control system. J. Huazhong Univ. of Sci.\&Tech, 2010.5, PP:79-82

[2] I.E.D. Pickup;A.P. Russell. Analysis of frequency and amplitudemodulation in the stabilisation of permanent-magnet synchronous/stepping motors. IEE Proc. B Electr. Power Appl. UK -July 1992 -- Volume 139, Issue 4, p.336-346

[3] GONG Shi-hua; LI Bin; ZHU Guo-li. Motion Control Technologies for Large-scale Turn-table Based on the Nonlinear Characteristics, ELECTRIC DRIVE, 2010.7, PP42-49. 